\title{
SPICE-Circuit Simulation of the Electrical Response of a Semiconductor Membrane to a Single-Stranded DNA Translocating Through a Nanopore
}

\author{
Amandine Leroux, Jacques Destiné, Benoît Vanderheyden, Maria E. Gracheva and \\ Jean-Pierre Leburton
}

\begin{abstract}
We describe a circuit-element model for the electric detection of biomolecules in translocation through a nanopore in a SOS semiconductor membrane. The biomolecules are simulated as a superposition of individual charges moving through the nanopore and inducing a charge variation on the membrane electrodes that is modeled as a current source. The SOS membrane is discretized into interconnected elementary circuit elements. The model is tested on the translocation of 11 base single-stranded C3AC7 DNA molecule, for which the electric signal shows good qualitative agreement with the multi-scale device approach of Gracheva et al., while quantifying the low-pass filtering in the membrane. Overall, the model confirms the possibility of identifying electrically the sequence of the DNA bases.
\end{abstract}

Keywords - Circuit modeling, DNA sequencing, solid-state nanopore, SPICE simulation 